\title{
Silver nanoparticle impregnated polycarbonate substrates for plasmonic applications
}

\author{
L. Lagonigro, ${ }^{1}$ T. Hasell, ${ }^{2}$ S. Rohrmoser, ${ }^{3}$ S. M. Howdle, ${ }^{2}$ P.J.A. Sazio, ${ }^{1}$ P.G. Lagoudakis, ${ }^{3}$ A.C. Peacock ${ }^{1}$ \\ ${ }^{1}$ Optoelectronics Research Centre, University of Southampton, Southampton SO17 1BJ, UK \\ ${ }^{2}$ School of Chemistry, University of Nottingham, NG7 2RD, UK \\ ${ }^{3}$ School of Physics and Astronomy, University of Southampton, SO17 1BJ, UK \\ e-mail: acp@orc.soton.ac.uk
}

\begin{abstract}
We present a new class of plasmonic substrates where silver nanoparticles are impregnated into a polycarbonate host. The substrates are shown to be exceptional candidates for SERS and metal enhanced fluorescence applications.
\end{abstract}

\section{INTRODUCTION}

Novel substrates based on noble metal nanoparticles are currently the subject of extensive research in fields such as biological sensing, medicine, spectroscopy and nano-photonics due to the large electromagnetic fields generated in the vicinity of the metal surface via a surface plasmon resonance [1]. Here we report on a new class of plasmonic substrate where silver nanoparticles are impregnated into a polycarbonate host using a high-pressure processing technique. The substrates are shown to be exceptional candidates for SERS and metal enhanced fluorescence (MEF) applications offering a number of advantages over existing substrates in that they are cheap, can be easily processed, and are bio-compatible. Furthermore, the embedding of silver nanoparticles into polymer substrates offers substantial environmental protection, allowing for the construction of temporally stable devices that can exploit the mechanical flexibility of the polymers.

\section{Substrate Characterization}

Fabrication of the substrates is based on supercritical carbon dioxide processing to produce silver nanoparticles in situ within prefabricated optically transparent polycarbonate strips [2]. The substrates have been characterized by energy dispersive $\mathrm{x}$-ray (EDX) analysis, transmission electron microscopy (TEM) and UV-vis absorption measurements. Fig. 1(a) shows the EDX spectrum which confirms the presence of metallic silver in the films. A TEM micrograph of a cross section of the silver-polycarbonate strip is shown in the inset revealing a nanoparticle band of uniform thickness $(\sim 6.5 \mu \mathrm{m})$ and composition along the length of the sample. A close up of the nanoparticles near the surface of the film is also provided as, owing to the highly localized electric field enhancements, it is these that are largely responsible for the plasmonic effects. From these images we estimate the size distribution of the nanoparticles to be $\sim 2-10 \mathrm{~nm}$. UV-vis absorption measurements of these substrates, plotted in Fig. 1(b), show a surface plasmon resonance around $410 \mathrm{~nm}$, in agreement with calculations based on Mie scattering theory for particles
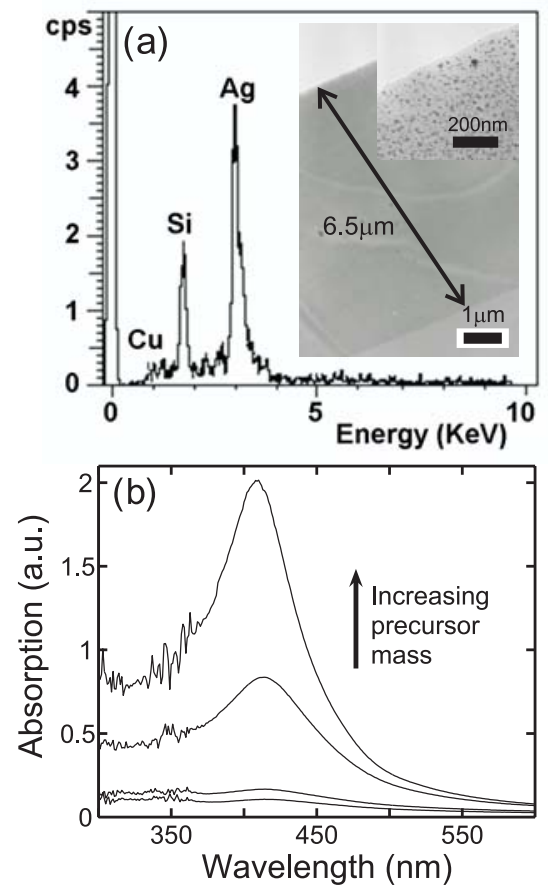

Fig. 1. (a) EDX spectrum of the nanoparticle band shown in the TEM micrograph of the AgPC substrate, plotted in the inset. Close up shows the surface particles. (b) UV-vis absorption spectra for various precursor concentrations.

with diameters $<10 \mathrm{~nm}$ embedded in a dielectric host with a refractive index of the pure polycarbonate. The intensity of the peak increases with the amount of precursor used, indicating an increase in the concentration of nanoparticles produced.

\section{SERS RESPONSE}

To investigate the SERS response of the metal-polymer composites, we drop cast $0.5 \mathrm{mM}$ of 4-aminothiophenol (4ATP) in ethanol onto the surfaces of both a silver impregnated (AgPC) and a pure (PC) polycarbonate substrate. The measured Raman spectra are plotted in Fig. 2(a) with the AgPC substrate (black line) clearly showing the main vibrational modes of 4-ATP, as assigned to those reported in the literature, whilst no Raman peaks were observed on the PC sample (grey line). SERS spectra collected a month after the 4-ATP deposition produced results of comparable intensity, 
confirming the temporal stability of the substrates. Further to this, we have also demonstrated the ability to post-process the films via simple oxygen plasma etching techniques, thus providing an additional degree of substrate design control. The measured SERS spectra on both the etched and the unetched regions of the sample are plotted in Fig. 2(b) with the etched profile shown in the inset (step of $\sim 2 \mu \mathrm{m}$ ). We attribute the enhanced SERS response on the etched region to an increase in the density of the nanoparticles going deeper into the film.
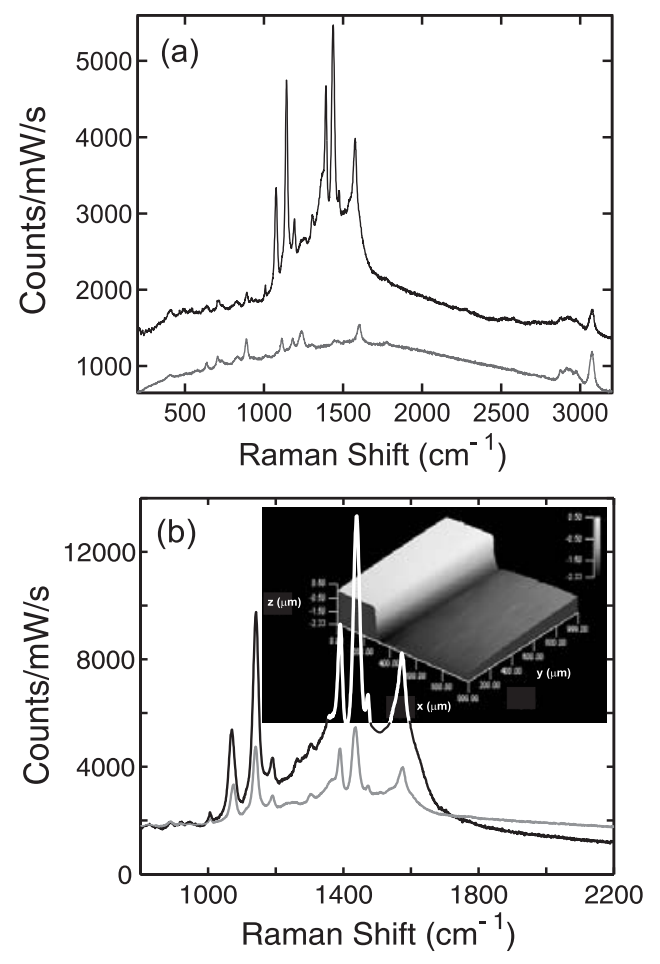

Fig. 2. (a) SERS spectra of 4-ATP deposited on AgPC (black line) and PC (grey line) substrates. (b) SERS spectra on the etched (black) and unetched (grey) regions of the substrate with a 3D profile image of the etched step shown in the inset.

\section{OBSERVATION OF MEF}

The metal-polymer composites were also tested for MEF using simultaneous time and spectral resolved measurements. We spin-coated a weak $0.5 \mathrm{mM}$ solution of Coumarin 102 in ethanol again onto both an $\mathrm{AgPC}$ and a PC substrate. The timespectral PL profiles obtained from the (a) PC and (b) AgPC substrates are plotted in Fig. 3, clearly showing an enhanced emission from the metal-polymer composite. From these we can obtain both the time integrated PL spectra (c) and the averaged PL decay curves (d), taken over the peak emission wavelength range $438-468 \mathrm{~nm}$. The enhanced fluorescence $(\sim 8.5 x)$, accompanied by a reduction in the photon lifetime, confirms the observation of MEF which is known to be associated with a change in the radiative decay rate [3].

\section{CONCLUSION}

Silver nanoparticle impregnated polycarbonate films have been demonstrated as unique substrates for plasmonic applications including routine SERS analysis and enhanced
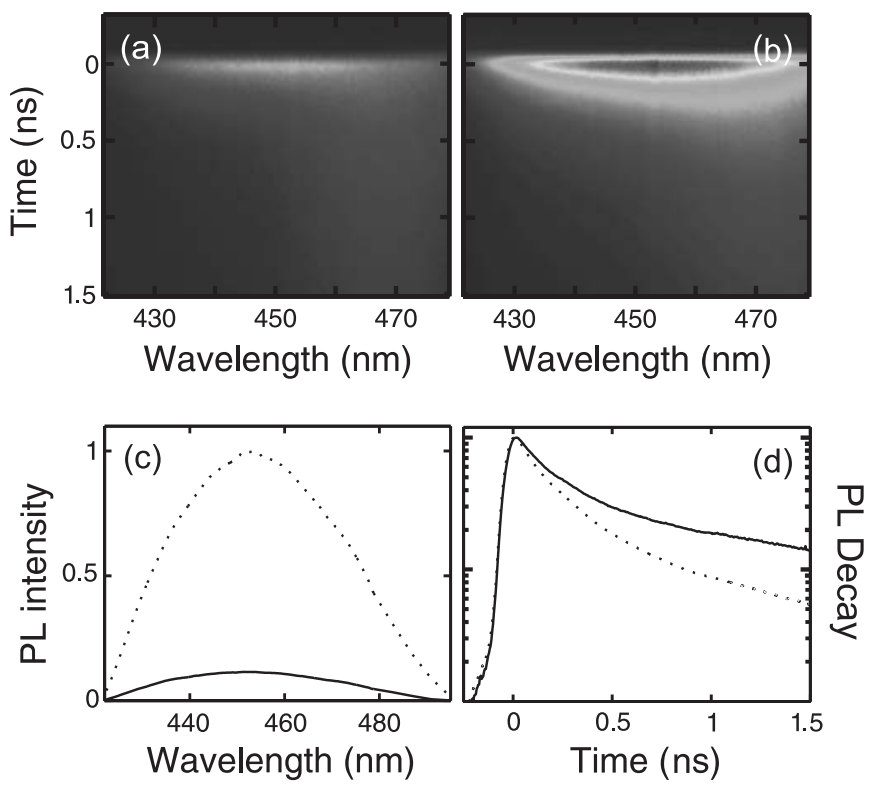

Fig. 3. (a) Time-spectral PL profiles for Coumarin on the (a) PC and (b) AgPC substrates. (c) Integrated PL spectra and (d) average PL decays for Coumarin on PC (solid line) and AgPC (dotted line) substrates.

fluorescence probes. Owing to the versatility of this highpressure particle impregnation technique, and its potential to be employed with a range of host polymer matrices and metal precursors, these substrates can be readily tailored for a wide range of applications in medicine and biology.

\section{ACKNOWLEDGMENT}

ACP acknowledges support from the Royal Academy of Engineering via a post-doctoral research fellowship.

\section{REFERENCES}

[1] E. Hutter and J. H. Fendler, "Exploitation of Localized Surface Plasmon Resonance," Adv. Mater. 16, 1685 (2004).

[2] T. Hasell, L. Lagonigro, A. C. Peacock, S. Yoda, P. D. Brown, P. J. A. Sazio, and S. M. Howdle, "Silver Nanoparticle Impregnated Polycarbonate Substrates for Surface Enhanced Raman Spectroscopy," Adv. Func. Mater. 18, 1265 (2008)

[3] J. R. Lakowicz, Y. Shen, S. D'Auria, J. Malicka, J. Fang, Z. Gryczynski, and I. Gryczynski, "Radiative Decay Engineering: 2. Effects of Silver Island Films on Fluorescence Intensity, Lifetimes, and Resonance Energy Transfer," Anal. Biochem. 301, 261 (2002). 